\title{
The role of haptic versus visual volume cues in the size-weight illusion
}

\author{
R. R. ELLIS and S. J. LEDERMAN \\ Queen's University at Kingston, Ontario, Canada
}

\begin{abstract}
Three experiments establish the size-weight illusion as a primarily haptic phenomenon, despite its having been more traditionally considered an example of vision influencing haptic processing. Experiment 1 documents, across a broad range of stimulus weights and volumes, the existence of a purely haptic size-weight illusion, equal in strength to the traditional illusion. Experiment 2 demonstrates that haptic volume cues are both sufficient and necessary for a full-strength illusion. In contrast, visual volume cues are merely sufficient, and produce a relatively weaker effect. Experiment 3 establishes that congenitally blind subjects experience an effect as powerful as that of blindfolded sighted observers, thus demonstrating that visual imagery is also unnecessary for a robust size-weight illusion. The results are discussed in terms of their implications for both sensory and cognitive theories of the size-weight illusion. Applications of this work to a human factors design and to sensor-based systems for robotic manipulation are also briefly considered.
\end{abstract}

In experimental psychology, weight perception can be traced back to the early experiments of Ernst Weber $(1834 / 1978)$. His main interest was whether weight perception resulted more from cutaneous inputs alone or from the muscular sense associated with lifting an object. He found that weight discrimination is more exact if the object is actually lifted rather than simply placed on a hand passively resting on a table. Since these early experiments, weight perception has been and continues to be investigated in many psychophysical experiments (see recent review by Jones, 1986). Despite this extensive research, it remains a problem for perceptual theorists.

Weight is an important dimension of an object, particularly if it has to be moved or manipulated by either a human or a robot. Its assessment presents an interesting paradox to a manipulator-in order to manipulate an object efficiently, its weight must be considered; however, to judge its weight, the object must be lifted. One cautious solution would be to try to lift the object with a small lifting force. If this force proved to be ineffective, it could be increased slightly. If this too proved to be unsuccessful, it could be increased repeatedly until an effective force was found that just lifted the object.

A more efficient solution to this paradox would be to use knowledge of particular objects and their properties derived from past experience for an initial weight esti-

This research was supported by a contract to $S$. J. Lederman by the Manufacturing Research Corporation of Ontario Centre of Excellence, and by a Postgraduate Level Scholarship from the Natural Sciences and Engineering Research Council of Canada to R. R. Ellis. The research fulfilled part of the requirements for a master's degree (Ellis, 1990). We would particularly like to express our appreciation to the Montreal Association for the Blind (Paul Barber was especially helpful in assisting us) and to the subjects who participated in Experiment 3. We also thank Cheryl Wilson. Reprint requests should be sent to S. J. Lederman, Psychology Department, Queen's University, Kingston, Ontario K7L 3N6, Canada. mate. This solution is limited to the extent that it would only succeed with objects that the manipulator had previously encountered.

A third and more universal solution would be to take advantage of the correlation between weight and volume, although no published research on this topic has been located. Large objects (particularly in the natural environment) tend to weigh more than small objects. Volumetric information can be quickly and easily processed without physically moving the object. Because a correlation between volume and weight is likely, knowing an object's volume provides coarse predictive information about its probable weight. It could, however, provide some surprising results in situations in which this natural correlation is violated. As humans move from terrestrial environments, where g-forces are quite constant, to either aquatic environments or outer space, where g-forces vary, the relationship between volume and weight becomes more uncertain, although the mass remains constant.

Charpentier (1891) first demonstrated that the perceived weight of an object, commonly referred to as its heaviness, depends not only on its physical weight but also on its size. He presented observers, who were allowed vision, with two spheres $(40 \mathrm{~mm}$ and $100 \mathrm{~mm}$ in diameter) of identical weight and had them lift each with the palm of their hand. Hand movements were not specified, but it is reasonable to assume that rather than keeping their palms rigidly flat, the observers cupped their hands to obtain volumetric information haptically as well as visually. The larger sphere was consistently reported as lighter. This phenomenon became known as the size-weight illusion. Flournoy's (1894) experiments extended the range over which the illusion occurred and also demonstrated that the illusion was so compelling that it persevered even when the observers were told that all the objects weighed the same. 
Many theories have attempted to explain the size-weight illusion. Early researchers regarded this phenomenon as a reflection of a density-constancy process (Thouless, 1931). Unfortunately, although an object's density (i.e., mass [or weight, if on earth]/volume) is undoubtedly involved, this concept of density-constancy does not explain any of the underlying mechanisms responsible for the observed phenomenon.

Another explanation involves the expectation theory (Ross, 1969), which states that prior experience with objects leads observers to expect that a larger object will be heavier than a smaller object. The correlation between large volumes and heavy weights would be high for comparisons within object sets that are either all solid or all uniformly hollow and made of the same (or very similar) materials. In cross-set comparisons, or when the objects are made up of radically different materials (i.e., sponge and granite), the correlation would be lower. However, these cases would represent exceptions, and in many instances would be artificial or man-made. Overall, particularly in natural environments, it is reasonable to assume that there is likely a somewhat significant correlation between large volumes and heavy weights. This learned correlation results in an expectation or mental set that could affect the force an observer applies when lifting an object.

A series of experiments by Davis and Roberts (1976) supports this cognitive theory. They found that when objects of identical weight are lifted, the larger objects are lifted with greater force, and therefore more quickly. Because it is assumed that subjects would attempt to lift all objects at the same rate, the greater velocity, acceleration, and deceleration found during the lift phase probably reflect the fact that the observers expected the larger object to weigh more. Therefore, they applied a greater lifting force to this object than to the smaller object, and because the lifting movement was faster, it was judged to be lighter.

A more perceptual theory that attempts to explain the size-weight illusion is derived from Anderson's (1970) "'information-integration' model. This theory, expanded by Cross and Rotkin (1975), considers size to be an object property that affects its perceived heaviness. From this perspective, the size-weight illusion is not an illusion at all; rather, it dramatically demonstrates that perceived heaviness is a function of both weight and volume. This interaction merely reflects an operating characteristic of the perceptual system involved, and no higher level process is required. This line of reasoning is not unprecedented. In audition, loudness is influenced by both frequency and sound pressure. Similarly, in vision, perceived hue is a product of both spectral wavelength and intensity.

Regardless of theoretical orientation, all the experimenters who have investigated the size-weight illusion (with the exception of Pick \& Pick, 1967) have confounded object volume (size) information extracted visually with that extracted haptically. In the standard task, we noie that the observers are always asked to judge an ob- ject's weight after they have picked up the object or after it has been placed in their hand. Lederman and Klatzky (1987) have shown that the stereotypical hand movement patterns or "exploratory procedures," called enclosure and unsupported holding (or grasp and lift, respectively), both provide object information sufficient for observers to perform a volume-matching task at above-chance levels. Enclosure is a hand movement in which the hand maximizes contact with as much of the object as possible by molding to its contours. Unsupported holding is a hand movement in which an object is lifted away from a supporting surface. Because one or both of these hand movements is normally used to lift an object, haptically derived volumetric information is clearly also available in the traditional method of assessing weight. Therefore, the observers in the traditional size-weight illusion experiments received volumetric information both visually and haptically when asked to make their weight estimates. This paper addresses the relative contributions of visual and haptic size information to the size-weight illusion.

Pick and Pick (1967) performed a developmental study of the size-weight illusion in which weight estimates of objects varying in volume were made haptically, visually, or by using haptics and vision together (the traditional method of assessing the size-weight illusion). They found that, although the magnitude of the traditional size-weight illusion remained stable with age, the magnitudes obtained in the other sensory conditions did not. Susceptibility to the illusion in the visual condition was initially low, and actually decreased as a function of age. The magnitude of the illusion derived solely through haptic inputs was also low in young children; however, it increased as a function of age, attaining and maintaining the same strength as that obtained in the visual + haptic condition by around 12 or 13 years. It is surprising that this interesting discovery concerning the contribution of haptically derived size information to the traditional size-weight illusion was not emphasized in their paper; for whatever reason, it has received no further attention in the literature to date.

Unfortunately, Pick and Pick (1967) did not ask their subjects to estimate the volume of the stimulus objects, so it is not possible to directly assess either the extent to which haptically versus visually derived estimates of size were comparable or the contribution of these judgments to apparent weight. Earlier, Koseleff (1957) used optically distorting lenses and Müller-Lyer patterns to demonstrate that perceived size does influence weight estimates.

A recent study by Masin and Crestoni (1988) required observers to estimate the weight of objects by pulling down on a ring attached to one end of a string. The string was fed through a pulley and its other end was attached to the object. In this way, the haptic volume information normally obtained by a grasp (i.e., via cutaneous and kinesthetic information to the fingers) was eliminated. One group was permitted vision, and another was not; the size-weight illusion occurred only when vision was allowed. It is interesting that it was essential that the object 
be viewed at the same time it was lifted. A third group looked at an object that was subsequently hidden from view, and then lifted it via strings; under such circumstances, there was no illusion. Masin and Crestoni used these results to refute the notion of cognitive expectation or motor set as the mechanism underlying the size-weight illusion, reasoning that any expectation should persist after the time the object is seen.

Masin and Crestoni (1988) therefore concluded that the size-weight illusion was a consequence of direct sensory origins. Unfortunately, they ran no observers in a standard paradigm (haptics + vision). As such, they had no baseline measure that could be used to compare the strength of the traditional illusion with the strength of the vision-only illusion they obtained. It may well be that the illusion they obtained was much weaker than a standard illusion and similar in strength to the visual illusion of Pick and Pick (1967) or to the color-weight illusion reported by DeCamp (1917). Nevertheless, the fact that the vision-only group showed any illusion indicates that vision is sufficient for the occurrence of a size-weight illusion.

This paper describes three experiments that were designed to assess the extent to which haptic and visual volume cues influence weight perception. In brief, Experiment 1 was conducted to determine if haptic volume cues alone could induce a size-weight illusion across a considerably more extended range of weights and volumes than those utilized by Pick and Pick (1967). After this was demonstrated, we assessed, in Experiment 2, the relative strengths of the size-weight illusion induced by each of the following four conditions: volume cues were obtained both visually and haptically, haptically only, visually only, or there were no volume cues available. Experiment 3 was designed to assess the necessity of visual mediation for the haptic size-weight illusion.

\section{EXPERIMENT 1 Haptics + Vision Versus Haptics}

This experiment was designed to assess the extent to which a purely haptically derived size-weight illusion could be produced using a range of volume and weight values that extended well beyond those used by Pick and Pick (1967). The strength of a resulting illusion would be compared with the strength of the illusion obtained using the traditional paradigm.

The ranges of weights and volumes used by Pick and Pick (1967) were 20-130 g and 39-100 cc, respectively. This study varied the stimuli across a broader range of volumes (149-8,615 cc) and weights (140-904 g), many of which required the use of two hands.

In the haptics + vision condition, observers were allowed to see the object while simultaneously picking it up to assess its heaviness. This traditional condition provided baseline data across a broad range of weights and volumes with which to compare the strength of any effects recorded in a haptics-only condition.
The haptics-only condition examined whether or not the visual system is necessary for the size-weight illusion. Observers were blindfolded and instructed to make haptic weight estimations of the stimuli. A significant size effect in this condition would indicate that visual processing is not necessary for the size-weight illusion. Furthermore, any significant difference between these results and the results of the haptics + vision condition would reflect the extent to which vision affects the illusion. No difference would suggest that visually processed volumetric information is of little importance to the illusion. On the other hand, if no illusion were demonstrated under this condition, it could be concluded that haptically processed volume information is not relevant or sufficient to the formation of the illusion.

\section{Method}

Stimuli. The stimuli consisted of three sets of cubes. Of these three sets, two consisted of seven different volumes. The volumes for each of these two sets were 149, 493, 1,093, 2,097, 3,652, 5,832 , and $8,615 \mathrm{cc}$. One set was fixed at $350 \mathrm{~g}$ and the other was fixed at $904 \mathrm{~g}$. The third set $(140 \mathrm{~g})$ was truncated after the first four volumes because it was not possible to produce the three larger sizes at this weight. These weights and volumes were chosen in order to represent the range of objects normally encountered in everyday situations (excluding the very small or very light values used by Pick \& Pick, 1967). There was also a single standard object of $1,093 \mathrm{cc}$ and $350 \mathrm{~g}$. All 19 cubes were covered in oak veneer; the edges of each cube were protected by strips of clear plastic wallpaper edging. The interiors of the cubes consisted of a mixture of metal and styrofoam that varied in accordance with the weight requirements. In all cases, the objects' weights were balanced about their centroids (centers of volume).

Observers. Sixteen ( 9 female, 7 male) paid university students ( 8 per condition) served as observers. They were experimentally naive, had normal or optically corrected vision, and had no known muscular or cutaneous problems. Their ages ranged between 17 and 40 years.

Procedure. The observers were randomly assigned to either the haptics + vision or the haptics-only condition. Those in the hapticsonly group were blindfolded. ${ }^{1}$ The standard object was placed on a table in front of the subjects and they were instructed to lift it and assign to it a weight of 100 units. They were also told that they would be given a set of objects and that their task would be to give numerical estimates (in decimals, fractions, or whole numbers) of weight relative to the standard. A magnitude estimate of weight was obtained on each trial. The subjects were given free access to the standard object throughout the experiment. Lifting the standard was mandatory before each presentation of a new object to minimize memory demands. No restrictions were imposed on hand movements prior to lifting, but only one-handed hefting was permitted. No feedback was provided between trials. Two blocks of the 18 test objects were presented to the observers, with order of presentation randomized within each block. Another block of the 18 objects was then presented to the observers and their magnitude estimates of volume were obtained.

\section{Results}

The arithmetic mean of the two estimates of weight given for each object was calculated for each observer. ${ }^{2}$ These means, the magnitude estimates of volume, the physical weight, and the physical volume were converted to common logarithms (base 10). Table 1 shows the sim- 
Table 1

Intercorrelation Matrices of $\log _{10}$ Transformed

Data for the 8 Observers in the Haptics-Only and Haptics + Vision Conditions in Experiment 1

\begin{tabular}{|c|c|c|c|c|}
\hline & $\begin{array}{l}\text { Physical } \\
\text { Weight }\end{array}$ & $\begin{array}{l}\text { Physical } \\
\text { Volume }\end{array}$ & $\begin{array}{l}\text { Perceived } \\
\text { Volume }\end{array}$ & $\begin{array}{l}\text { Perceived } \\
\text { Weight }\end{array}$ \\
\hline \multicolumn{5}{|c|}{ Haptics Only } \\
\hline $\begin{array}{l}\text { Physical Weight } \\
\text { Physical Volume } \\
\text { Perceived Volume } \\
\text { Perceived Weight }\end{array}$ & & 0.24 & $\begin{array}{l}0.25 \\
0.95\end{array}$ & $\begin{array}{r}0.85 \\
-0.20 \\
-0.17\end{array}$ \\
\hline \multicolumn{5}{|c|}{ Haptics + Vision } \\
\hline $\begin{array}{l}\text { Physical Weight } \\
\text { Physical Volume } \\
\text { Perceived Volume } \\
\text { Perceived Weight }\end{array}$ & & 0.24 & $\begin{array}{l}0.23 \\
0.96\end{array}$ & $\begin{array}{r}0.79 \\
-0.25 \\
-0.24\end{array}$ \\
\hline
\end{tabular}

ple intercorrelation (Pearson product-moment) matrix of these four factors under both conditions.

These matrices reveal that for both the haptics-only and haptics + vision conditions, the best predictor of estimated weight is physical weight, $r=.85$ and .79 , respectively. Again for both conditions, the next best predictor is physical volume, $r=-.20$ and -.25 , respectively. Due to the high correlation between perceived volume and physical volume ( 0.95 and 0.96 , respectively), perceived volume was dropped from further analysis and is addressed in the General Discussion. A forward selection multiple regression analysis was performed on the $\log _{10}$ transforms of the remaining three factors under both conditions in order to determine how well physical weight and physical volume predicted the observers' estimates of weight. The mean regression equation for the hapticsonly condition is:

$$
y=.26+.98 x-.23 z
$$

where $y=\log _{10}$ estimated weight, $x=\log _{10}$ physical weight, and $z=\log _{10}$ physical volume $\left[r^{2}=.89\right.$, $F(1,141)=554.15, p<.0001]$.

The corresponding regression equation for the haptics + vision condition is:

$$
y=-.09+1.25 x-.35 z
$$

$\left[r^{2}=.82, F(1,141)=327.37, p<.0001\right)$.

The results of this analysis show that for haptics only and haptics + vision, physical weight is the best predictor of estimated weight (partial $r^{2}=.72$ and .62 , respectively, $p<.0001$ ). This was followed by physical volume (partial $r^{2}=.17$ and .20 , respectively, $p<.0001$ ).

A simple regression analysis of $\log _{10}$ estimated weight versus $\log _{10}$ physical volume was performed for each observer at each weight level. A one-within (weight, three levels), one-between (modality, two levels) analysis of variance (ANOVA) performed on the slopes of the resulting regression lines revealed that none of the factors or their interaction was statistically significant. Since the slopes represent the strength of the illusion, neither weight level nor modality affected illusion strength.
A separate mixed two-factor design ANOVA (three levels within, two levels between) was also performed on the corresponding $y$ intercepts of the regression lines. This showed a significant effect of weight $[F(2,28)=27.97$, $p<.0001]$. No significant effects were found for either modality $[F(1,14)=3.23, p=.09]$ or for the interaction of modality and weight $[F(2,28)=0.51, p=.61]$. This indicates that an object's actual weight affects its perceived weight and that there were little or no modality differences in this perception.

Figure 1 shows these values plotted against $\log _{10}$ physical volume as well as the regression lines fitted to these data. The top pair of regression lines indicates the heaviness estimates for the 904-g set of objects, the middle pair indicates estimates for the $350-\mathrm{g}$ set of objects, and the lowest pair corresponds to weight estimates for the $140-\mathrm{g}$ object set. At the latter weight level, recall that there are only four physical volumes as opposed to the seven volumes found at the other two weight levels. The two regression lines making up each pair represent the hapticsonly and haptics + vision conditions. Although the slopes of these six regression lines are not significantly different, it can be seen that at each weight level the regression line for the haptics + vision condition is slightly steeper than that obtained in the haptics-only condition.

\section{Discussion}

The results of this experiment extend Pick and Pick's (1967) finding that strong size-weight illusions can be ob-

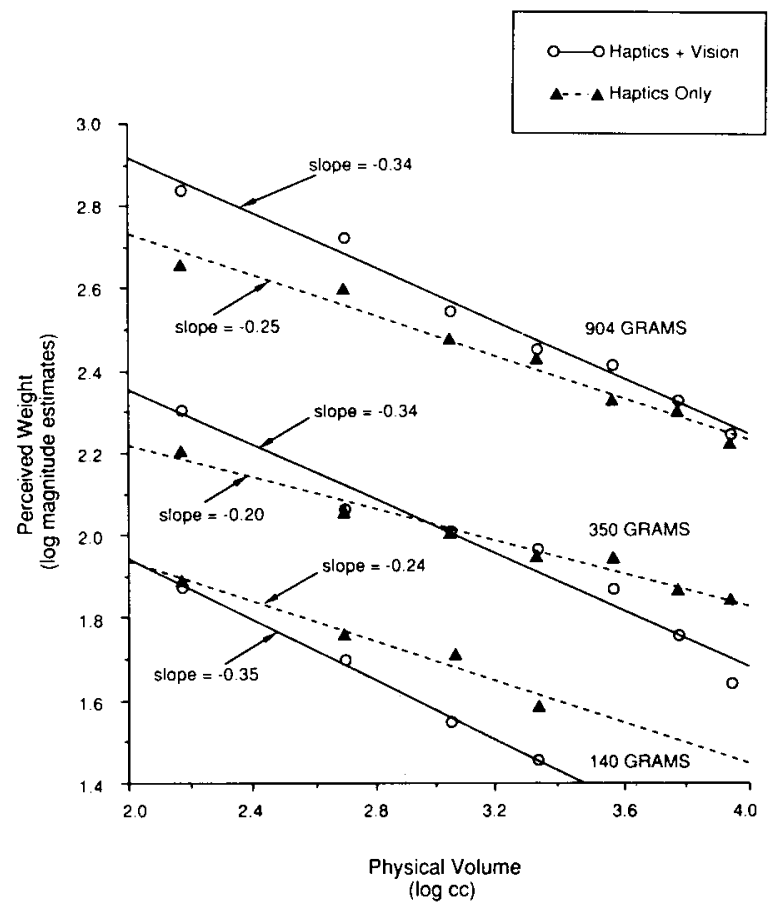

Figure 1. $\log _{10}$ (mean magnitude estimates of weight) as a function of $\log _{10}$ physical volume for modality (haptics + vision; haptics only) and weight level in Experiment 1. Each data point is based on eight scores. 
tained when only the haptic system is permitted to obtain volumetric information. The strengths of these illusions were not significantly different from those obtained from observers who were allowed both haptic and visual volume cues.

This finding was consistent across the broad range of weights (140-904 g) and volumes (149-8,615 cc). Not only do the slopes of the regression lines not differ significantly between conditions at each weight level, but there also is no significant difference in slope for each condition between weight levels. This indicates that weight, at least within the range used in this experiment, does not affect the strength of an observer's size-weight illusion.

It is not surprising that weight has a significant effect on the $y$ intercepts of the lines. This merely reflects the fact that heavier objects of the same volume were assigned higher magnitude estimates of weight than were lower weighted objects. This mirrors the results of the multiple regression analysis and indicates that physical weight has a strong effect on perceived weight.

The powerful size-weight illusion produced in the haptics-only condition suggests that the direct use of vision is not a necessary condition for the formation of a size-weight illusion comparable in strength to the traditional illusion. Any comprehensive theory attempting to explain the illusion must account for this fact; the contention that the visual system dominates or influences haptic weight perception in a "visual capture" sense must be reevaluated.

\section{EXPERIMENT 2 Visual Versus Haptic Contribution}

Experiment 1 showed that the greatest difference in illusion strengths between the haptics-only and haptics + vision conditions occurred at the middle weight level of $350 \mathrm{~g}$. The difference in slopes resulting from these two conditions, although not significant, is large enough to question whether excessive variability is masking a genuine effect. Therefore, this weight level was chosen to be the stimulus value for a more comprehensive study aimed at evaluating the relative contributions of both visual and haptic size information. To decrease the variability of this experiment in comparison with Experiment 1 , a sample size of 24 observers was used in a within-subjects design.

Experiment 1 demonstrated that vision is not necessary for a size-weight illusion. But is vision alone sufficient for the illusion? The study by Masin and Crestoni (1988) confirms that this is so, but fails to address whether its magnitude is equivalent to the standard size-weight illusion. Pick and Pick (1967) also confirm the existence of a visual size-weight illusion for relatively small, lightweight objects; their data further indicate that the illusion is much weaker than the traditional one.

To determine the relative roles of each source of sensory input, the following four conditions were presented in random order to all participants. The first two modal- ity conditions (haptics + vision and haptics only) were identical to those used in Experiment 1. In the third condition (vision only), the observers were allowed only visual cues about object size but were, however, provided with kinesthetic cues for weight assessment. Pick and Pick (1967) and Masin and Crestoni (1988) did produce an effect with this general paradigm; we further consider its strength compared with that of the effects produced under the other conditions. A fourth condition, not used by Pick and Pick (no haptics or vision) denied observers both visual and haptic size information. Without size information, there should be no size-weight illusion. If an illusion does occur, it would suggest that some volume information was available to the observer. Thus, this condition was used to assess whether volume cues can be effectively eliminated.

\section{Method}

Stimuli. The stimuli in this experiment consisted of the same set of middle weight $(350 \mathrm{~g}$ ) objects, the same standard object, and the same modulus of 100 that was utilized in Experiment 1 .

Observers. Twenty-four (12 male, 12 female) observers from Experiment 1 were used in this experiment.

Procedure. Each observer was randomly assigned to one of the 24 permutations of the four modality conditions. The standard cube was placed in front of the observers and they were given the instructions that were used in Experiment 1 . In conditions in which vision was not permitted, they were blindfolded. In the haptics + vision and haptics-only conditions, the procedure from Experiment 1 was used. In the third condition (vision only), the observers were not allowed to directly handle either the comparison objects or the standard. Rather, their weight estimates were based on their lifting strings from which the cubes were suspended. During this procedure, the cubes were placed in a wooden framework that constrained them from swinging sideways or spinning, thus allowing only vertical movements. Note that this differs from the vision-only condition used by Masin and Crestoni (1988) in that we used no rings or pulleys and our subjects lifted up, whereas theirs pulled down. The observers in the fourth condition, no haptics or vision, followed the same procedure as the vision-only subjects, but they were blindfolded.

Within each condition, the set of seven test objects was placed one at a time in front of the observers, and magnitude estimates of weight were solicited. No feedback was provided, and in all conditions the observers were allowed free access to the standard object throughout the experiment. It was mandatory that the standard be lifted before each presentation of a new test object.

\section{Results}

Mean regression lines (collapsed across the 24 observers) of $\log _{10}$ weight estimates on $\log _{10}$ physical volumes were calculated under each modality condition. These are shown, with their slopes, in Figure 2.

As shown in Figure 2, the steepest slope was obtained in the haptics-only group. The mean regression line for this group indicates that a doubling of physical volume resulted in a $26 \%$ decrease in an observer's estimate of weight. The coefficient of determination $\left(r^{2}\right)$ for this regression is .987 .

The haptics + vision condition had a slightly shallower slope. Its regression line denotes that, in this condition, a doubling of physical volume resulted in a $24 \%$ lower- 
ing of an observer's weight estimate. The coefficient of determination for this regression line is $\mathbf{9 8 5}$.

The next steepest average slope was obtained in the vision-only condition. This group's regression line shows that when the physical volume was doubled, the resulting magnitude estimation of weight was reduced by $17 \%$. The coefficient of determination for this regression line is .996 .

The last condition, no haptics or vision, produced a flat function. The mean regression line denotes that a doubling of physical volume resulted in only a $0.3 \%$ decrease in the observer's estimate of weight. As expected with a slope close to zero, the coefficient of determination for this regression line is very low, .018 .

The individual slopes were used in a repeated measures one-way ANOVA, which revealed a significant effect of modality $[F(3,69)=49.88, p<.0001]$. Nonorthogonal tests of the individual modality conditions indicate that the haptics-only condition had a significantly steeper slope than the vision-only condition $[F(1,23)=15.41, p=$ $.0007]$ and the haptics + vision condition also had a significantly steeper slope than the vision-only condition $[F(1,23)=13.69, p=.0012]$. There was no significant difference between the haptics-only and haptics + vision conditions $[F(1,23)=2.53, p=.13]$. The standard error of the slopes was used to compute confidence intervals for each condition to ensure that a genuine illusion had occurred (i.e., a nonzero slope). As shown in Table 2, these intervals at $p=.001$ for the haptics-only, haptics + vision, and vision-only conditions did not include zero, and thus all had significant negative slopes. The slope ob-

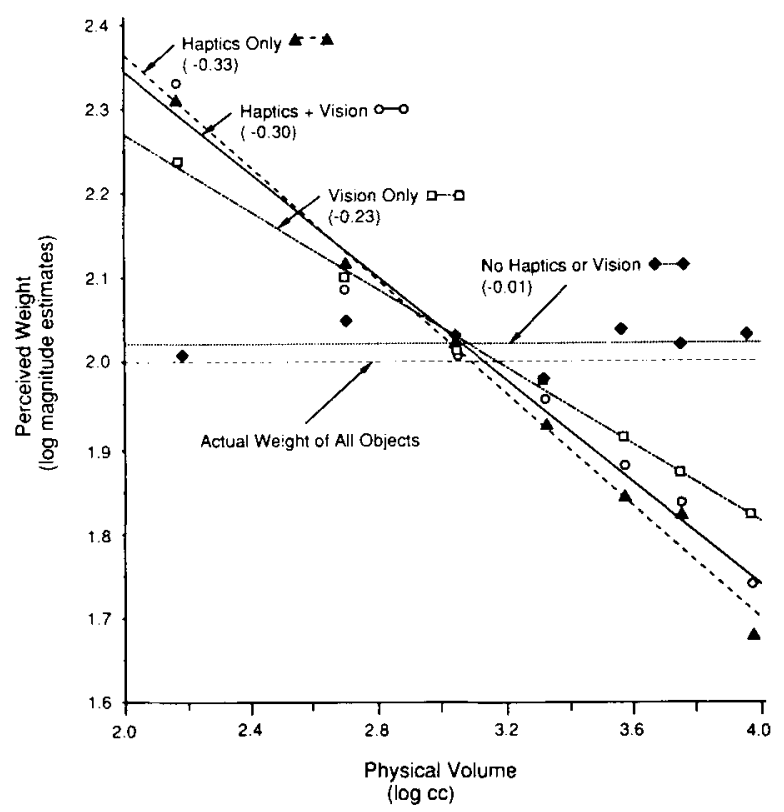

Figure 2. $\log _{10}$ (mean magnitude estimates of weight) as a function of $\log _{10}$ physical volume for each modality in Experiment 2. Each datum is based on 24 scores. The dotted line indicates a veridical heaviness estimate. Slopes are shown in parentheses.
Table 2

Confidence Intervals for the Slopes of the Simple Regressions of $\log _{10}$ (Estimated Weight) on $\log _{10}$ (Physical Volume) by Condition in Experiment 2

\begin{tabular}{lc}
\hline \multicolumn{1}{c}{ Condition } & Confidence Interval \\
\hline Haptics Only & -0.2185 to $-0.4459^{*}$ \\
Haptics + Vision & -0.1806 to $-0.4180^{*}$ \\
Vision Only & -0.1361 to $-0.2384^{*}$ \\
No Haptics or Vision & -0.0210 to $+0.0175 \dagger$ \\
\hline
\end{tabular}

${ }^{*} p=.001 . \quad \dagger p=.05$.

tained in the no-haptics-or-vision condition was not significantly different from zero.

\section{Discussion}

Confidence intervals performed on the mean of the resulting slopes of the regression lines for each of the four groups clearly show that a significant size-weight illusion was obtained in the haptics + vision, haptics-only, and vision-only conditions. The high coefficients of determination demonstrate that $\log _{10}$ physical volume is a good predictor of estimated weight and that the function is highly linear, indicating that heaviness is a power function of physical volume.

As hypothesized, the confidence interval for the slope of the regression equation of $\log _{10}$ estimated weight against $\log _{10}$ physical volume shows that the size-weight illusion was not generated under the no-haptics-or-vision condition. This is further indicated by the low coefficient of determination for the mean regression line shown in Figure 2 . This demonstrates that no significant volume cues were transmitted to the observers either visually through the blindfold, haptically through the string (moment of inertia), or audibly through noises generated while handling the objects. It also indicates that in the haptics-only condition, haptic cues were the only significant source of volume information (i.e., the blindfold prevented cues). Similarly, in the vision-only condition, visual cues were the only source of volume information.

The results of the ANOVA performed on the slopes of the observers' individual regression equations confirm the hypothesis that a size-weight illusion produced when object volume information is conveyed by haptics only is just as powerful as when volume information is conveyed using the traditional haptics + vision paradigm. This statistical equivalence is in complete agreement with the results of Experiment 1, despite the greater sensitivity of this experiment.

The illusion produced in the vision-only condition was significantly less substantial than that produced in either the haptics + vision or haptics-only conditions. These results indicate that, although both haptic volume cues and visual cues are sufficient to produce a size-weight illusion, it is the haptic rather than the visual cues that are necessary to generate a full-strength illusion. The theoretical implications of this finding will be discussed in the General Discussion. 


\section{EXPERIMENT 3 Congenitally Blind Subjects}

Although Experiments 1 and 2 demonstrated a compelling haptic size-weight illusion in the absence of visual cues, this does not necessarily indicate that the visual system is uninvolved. It may be that the subjects translated the haptic inputs they received into a visual image, which was then reperceived through visual processors. Such a model of haptic processing has frequently been proposed (e.g., see the "image-mediation" model discussed in Klatzky \& Lederman, 1987).

An alternative to this visual mediation model is the "direct haptic apprehension" model, which views haptics as a separate processing system (Klatzky \& Lederman, 1987). According to this model, information about objects and surfaces is extracted by haptic sensors and is processed haptically to form haptic object representations. Such representations and the processes used to derive them are subject to specific constraints on the haptic system. In later stages, the visual and haptic representations may in fact be combined to produce an integrated representation, with the strengths of the visually and haptically derived inputs weighted by task constraints and the relative effectiveness with which a given property can be extracted.

To explore this issue in Experiment 3, we replicated the stimuli and methods of Experiment 2, but used different subject groups. One group consisted of 8 congenitally blind observers; the other was a control group of 8 sighted observers. Since the congenitally blind have never had any visual experience, it is very unlikely that they generate visual images. Therefore, if they experienced a strong illusion that was comparable to that experienced by the sighted group, such a result would provide converging evidence that vision is not necessary for the illusion. Furthermore, it would indicate that haptic volume information does not need to be translated into a visual image, thus supporting the direct haptic apprehension model of haptic processing.

This experiment had two modality conditions, both of which required the sighted participants to be blindfolded. The haptics-only condition was the same as that used in both Experiments 1 and 2. The no-haptics-or-vision condition was the same as that used in Experiment 2.

The results of Experiments 1 and 2 indicated that haptics only is a sufficient condition for a full-strength sizeweight illusion. Based on this finding, and in support of the direct haptic apprehension model of haptic processing, we hypothesized that congenitally blind observers would report a size-weight illusion as strong as that reported by blindfolded, sighted observers when provided with only haptic volume cues. It was further hypothesized that in the absence of volume cues, neither group would experience an illusion.

\section{Method}

Stimuli. The stimuli in this experiment consisted of the same set of cubes and wooden guides that were used in Experiment 2.
Observers. Sixteen experimentally naive, paid observers, aged 16-35 years, were used. Eight ( 5 female, 3 male) were university students who had normal or optically corrected vision. The other eight ( 5 male, 3 female), were congenitally blind observers recruited from the Montreal Association for the Blind. Of these, 5 had no vision and 3 could perceive light, but not patterns.

Procedure. The observers, each in turn, were blindfolded if sighted, and the procedures used in the haptics-only and no-hapticsor-vision conditions of Experiment 2 were followed. All the participants were exposed to both modality conditions; the order of these conditions was counterbalanced.

\section{Results}

The physical volumes of the test objects and the observers' magnitude estimates of weight were transformed logarithmically (base 10). Simple individual regression lines of $\log _{10}$ estimated weight $(y)$ on $\log _{10}$ physical volume $(x)$ were collapsed across the 8 observers and a mean regression line was calculated for each group under both conditions (see Figure 3).

The mean regression lines for the blind and sighted observers in the haptics-only condition have slopes of -.29 and -.27 , respectively. This indicates that a doubling of physical volume resulted in a $22 \%$ and $21 \%$ decrease in weight estimates, correspondingly. The coefficients of predictability for these regressions are .99 and .94 , respectively. A between-group $t$ test revealed no significant difference between the slopes of these two mean regression lines $[t(14)=.512, p=.62]$.

The last two slopes, representing the blind and sighted groups for the no-haptics-or-vision condition, are not significantly different from zero. The $y$ intercept of both these lines is not significantly different from 2 (common log of modulus). This indicates that, for both groups, all the objects were judged as weighing the same as the standard.

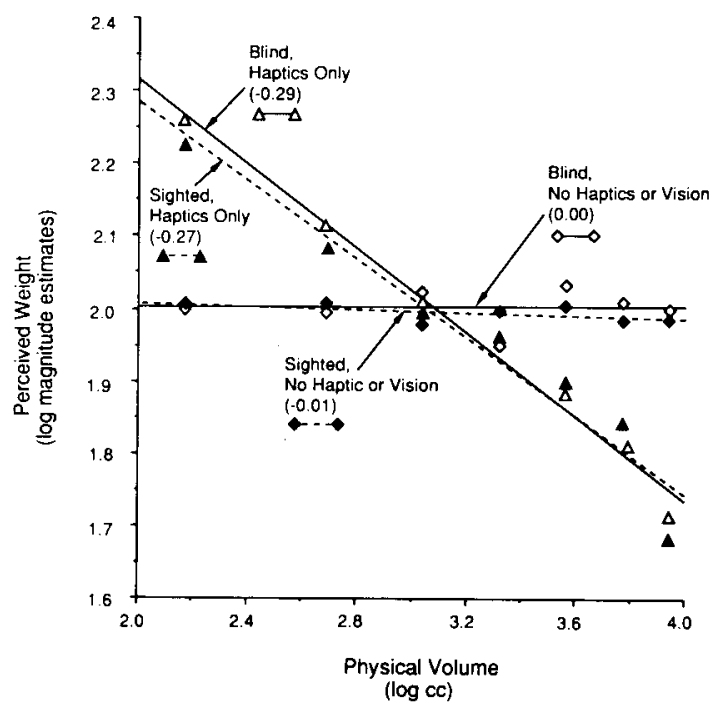

Figure 3. $\log _{10}$ (mean magnitude estimates of weight) as a function of $\log _{10}$ physical volume for modality (haptics only; no haptics or vision) and group (blind vs. sighted blindfolded) in Experiment 3. Each datum is based on eight scores. The slopes of the regression lines are shown in parentheses. 


\section{Discussion}

The results of Experiment 3 confirm the hypothesis that congenitally blind observers experience a size-weight illusion as compelling as that observed by blindfolded, sighted observers. This demonstrates that haptic volume information does not need to be translated into a visual image that is subsequently reanalyzed visually.

Rather, the fact that congenitally blind observers experience a full illusion adds converging evidence to the theory that the size-weight illusion is primarily a haptic phenomenon in which vision is unnecessary. It can, in fact, be generated totally via haptic processing, which lends support to the direct haptic apprehension model.

The hypothesized lack of a size-weight illusion in the no-haptics-or-vision condition in both blind and blindfolded, sighted observers was also confirmed. This control condition demonstrated that there were no extraneous sources of volume information (e.g., sound cues, moment of inertia) that could be used to judge weight. Thus, weight estimates recorded in the haptics-only condition were presumably based on the haptic information about the mass of the object.

\section{GENERAL DISCUSSION}

The results of Experiments 1-3 clearly confirm the existence of a robust haptic size-weight illusion, equal in strength to the traditional illusion reported when both haptic and visual volume cues are provided. This finding, together with the similarity in the standard errors of slope found in these conditions, strongly suggests that the traditional size-weight illusion is primarily a haptic phenomenon.

Experiment 1 documented the haptic size-weight illusion across a wide range of weights (140-904 g) and volumes $(149-8,615 \mathrm{cc})$. When the present results are considered with those obtained by Pick and Pick (1967), it can be seen that the illusion evidently occurs across the full range of weights and volumes encountered when manipulating objects with one or two hands. In Experiment 2 , we confirmed this finding with the $350-\mathrm{g}$ set by using a more sensitive procedure that involved a repeated measures design and a larger sample size. With the use of congenitally blind observers, we demonstrated in Experiment 3 that visual imagery is not a necessary component of the illusion either; thus, neither real vision nor visual imagery is apparently required. A size-weight illusion of traditional strength is generated when input is derived solely by the haptic system.

Conversely, the results of Experiment 2 showed that when only visual volume cues were available, that is, when haptic volume cues were denied, a size-weight illusion did occur; however, it was weaker than either the traditional or the haptic illusion. Magnitude estimates of perceived volume further indicated that this weaker illusion did not result from differences in perceived volume across modalities. Thus, haptic volume information is both necessary and sufficient for a full-strength illusion. In contrast, visual volume cues are sufficient to produce an illusion of lesser magnitude. These findings extend and help disambiguate those obtained by Pick and Pick (1967) and Masin and Crestoni (1988). Pick and Pick demonstrated the same pattern of results by using small light objects. However, they did not examine whether volume was estimated similarly by haptics and vision, nor did they include a control condition that eliminated all information about volume. This served to ensure that volumetric information, when available, was strictly accessible through vision or haptics in the vision-only and haptics-only conditions, respectively (i.e., it ensured that no haptic volumetric information could be conveyed through the string in the vision-only condition). Masin and Crestoni also demonstrated a vision-only size-weight illusion, but did not measure the traditional illusion for purposes of comparison. In Experiment 2 of the current paper, we found that the strength of the visual size-weight illusion was only $78 \%$ of the traditional illusion and $69 \%$ of the pure haptic illusion.

These findings indicate that weight estimates made when both visual and haptic size information is available are the same as those made when only haptic size cues are allowed. This occurs despite the more veridical weight estimates obtained when only visual size cues are allowed. This appears to be a situation in which touch educates vision, as Berkeley $(1709 / 1957)$ suggested, and thus could be used as an example of haptic capture in the same sense that Rock and Harris (1967) suggested visual capture (see review by Welch $\&$ Warren, 1981).

The present experiments do not strongly address the sensory versus cognitive bases of the illusion. The results do, however, tend to agree with Masin and Crestoni's (1988) theory that the sensory component is essential to the formation of a strong size-weight illusion. The modality differences found in these experiments present problems for the theoretical explanations for the size-weight illusion that were described in the introduction. The expectation theory (Ross, 1969) did not predict any difference between the magnitude of haptic versus visual size-weight illusions. This cognitive theory states that prior experience with objects results in a learned correlation between large volumes and heavy weights. Because the observers' estimates of volume were unaffected by the input modality, there is no reason to expect the strength of the illusion to differ with input modality.

Anderson's (1970) information-integration theory also seems unable to explain the results of these experiments. It states that perceived heaviness is a direct function of both weight and volume, so it is inappropriate to describe the pattern of results as a size-weight "illusion." In principle, this perceptual theory might explain the differences in the magnitude of the illusion by relating them to differences in the abilities of the input modalities to differentiate variations on the nontargeted dimension. However, the same basic question remains: If both modalities 
assess volume equally well (at least for these simple regular cubes), why should different input modalities produce unequal strengths?

One answer may lie in the fact that, when judging the weight of an object, the haptic system can directly sense the linear forces and torques (rotational forces) applied when the arm/hand lifts the object. Although the ecological approach argues that visual kinematic cues to dynamic events may also be used to assess lifted weight (e.g., Runeson \& Frykholm, 1981), it seems unlikely that the visual cues available to the observer about an object's weight in the vision-only condition would provide information as discriminating as that obtained when the observer grasps and lifts the object. It seems reasonable, therefore, to argue that weight-estimation tasks involving the normal grasp-and-lift cycle fall more naturally (although not exclusively) within the haptic domain. Under such circumstances, there would be no need to translate the volume information also used to estimate weight. Haptic volumetric information is simply and elegantly provided as a by-product of the exploratory procedures used to extract weight information.

However, when the only volume cues for judging weight are visual, there can only be a visual representation of volume. It may be the case that a visual representation loses some of its strength or relevance when used along with the haptic representation of weight as a factor in weight estimation. In effect, the connection between the haptic weight representation and the visual volume representation may be weaker than the connection between haptic weight and haptic volume representations.

Alternatively, the visual representation may have to be translated into a haptic representation before it can influence a subsequent weight estimate. This translation process may result in a haptic volume representation that is inferior to the one derived from manual exploration. Note that this argument is the antithesis of the image-mediated model of haptic processing, which has most often been applied to form perception tasks: with respect to weight estimation, it may be necessary for visual inputs to be translated into haptic representations for subsequent haptic processing.

\section{Observers' Estimates of Volume}

Koseleff (1957) demonstrated that when an object's perceived size is changed, either through the use of an optically distorting lens or with Müller-Lyer patterns, perceived weight also varies. When an object appears larger, it feels lighter; when it appears smaller, it feels heavier.

Across all the modality conditions and experiments reported in this paper, the observers provided magnitude estimates of volume for each object subsequent to their estimates of weight. However, these estimates of volume were never significantly different across modalities. Moreover, they were not better predictors of estimated weight than actual volume, given the very high correlation between physical volume and estimated volume. As a result, the effects reported here cannot be attributed to differences in apparent volume of the type recorded by Koseleff (1957).

For the purposes of this paper, and in keeping with the vast majority of researchers, the term size has been interpreted as physical volume. However, because all the objects were cubes, linear extent, area, and volume all covaried with size. Therefore, it cannot be shown that the observers used volume as a basis for weight judgment. A recent study by Summers (1991) demonstrated that when people were asked to judge a three-dimensional object's "size," the best linear fit to these data, using a $\log / \log$ coordinate system, was volume as opposed to onedimensional length or two-dimensional area. This problem in reference to the size-weight illusion is currently being investigated by the authors.

\section{Applications}

The results of the current study may be applied to two different problems. First, we note that volume could be an important factor in ergonomic considerations of weight or force manipulation. For example, Luczak and $\mathrm{Ge}$ (1989) point out that, although volume is an important consideration in the lifting capacity of humans, its influence is not considered in standards set to define maximum acceptable weights hefted in industrial lifting tasks. A further example (at more moderate weight levels) might be the size of a control lever's handle in a hydraulic system; its size could be an important ergonomic consideration for a human operator, because size could influence the estimation of an appropriate force required to move the lever in order to maintain a realistic "feel" to the control system.

Second, the current results suggest a general data-driven robotic strategy for lifting objects of unknown mass. Volumetric computations based on the initial grasp might be used to provide the first estimate of what is required to optimize the subsequent lift action. The cutaneous inputs from the mechanoreceptors and the dynamics of the subsequent arm movements could then be used to fine-tune the lift sequence. Presumably, the tactile/haptic cues would optimize the initial lift action, because the same effector configuration is employed during the entire perception/action cycle.

\section{Summary}

A set of three experiments provides compelling evidence that the traditional size-weight illusion is primarily a haptic phenomenon. Haptic volume cues alone were found to be both sufficient and necessary to generate a purely haptic size-weight illusion with a strength comparable to that of the traditional illusion produced when both vision and haptics are allowed.

Furthermore, congenitally blind participants demonstrated equally strong illusions, thus indicating that haptic volume information does not need to be transformed into visual images in order to be effectively processed. This supports Klatzky and Lederman's (1987) direct haptic apprehension model of haptic processing. 
Conversely, when volume information is obtained from the visual system only, the resulting size-weight illusion is significantly weaker. This occurs despite similar estimates of volume across modalities. This indicates that vision, although sufficient to induce a weaker illusion, is neither necessary nor sufficient to generate a size-weight illusion of traditional strength.

These findings suggest a sensory explanation for the size-weight illusion, and oppose exclusively cognitive theories that should be less influenced by the modality through which volume information is processed.

\section{REFERENCES}

Anderson, N. H. (1970). Averaging model applied to the size-weight illusion. Perception \& Psychophysics, 8, 1-4.

Berkeley, G. (1957). A new theory of vision. London: J. M. Dent \& Sons. (Original work published 1709)

Charpentier, A. (1891). Analyse experimentale de quelques elements de la sensation de poids [Experimental study of some aspects of weight perception]. Archives de Physiologie Normales et Pathologiques, 1.3, 122-135.

Cross, D. V., \& Rotkin, L. (1975). The relation between size and apparent heaviness. Perception \& Psychophysics, 18, 79-87.

DAvis, C. M., \& ROBERTS, W. (1976). Lifting movements in the sizeweight illusion. Perception \& Psychophysics, 20, 33-36.

DE CAMP, J. E. (1917). The influence of colour on apparent weight: A preliminary study. Joumal of Experimental Psychology, 2, 347-370.

FLournoy, T. (1894). De l'influence de la perception visuelle des corps sur leur poids apparent [The influence of visual perception on the apparent weight of objects]. L'Année Psychologique, 1, 198-208.

JoNEs, L. A. (1986). Perception of force and weight: Theory and research. Psychological Bulletin, 100(1), 29-42.

Klatzky, R. L., \& Lederman, S. J. (1987). The intelligent hand. In G. W. Bower (Ed.), The psychology of learning and motivation (Vol. 21, pp. 122-149). San Diego: Academic Press.

Kose LefF, P. (1957). Studies in the perception of heaviness. Acta Psychologica, 13, 242-252.

LEDERMAN, S. J., KlatZKY, R. L. (1987). Hand movements: A window into haptic object recognition. Cognitive Psychology, 19, 342-368.
LuCzak, H., \& GE, S. (1989). Fuzzy modelling of relations between physical weight and perceived heaviness: The effect of size-weight illusion in industrial lifting tasks. Ergonomics, 32, 823-837.

Masin, C. M., CRestoni, L. (1988). Experimental demonstration of the sensory basis of the size-weight illusion. Perception \& Psychophysics, 44, 309-312.

Pick, H. L., \& Pick, A. D. (1967). A developmental and analytic study of the size-weight illusion. Journal of Experimental Child Psychology, 5, 362-371.

Rock, I., Harris, C. S. (1967). Vision and touch. Scientific American, 216, 96-104.

Ross, H. E. (1969). When is weight not illusory? Quarterly Journal of Experimental Psychology, 21, 346-355.

Runeson, S., Frykholm, G. (1981). Visual perception of lifted weight. Journal of Experimental Psychology: Human Perception \& Performance, 7, 733-740.

SUMmers, C. (1991). Haptic exploration with and without vision: Property encoding and object representation. Dissertation Abstracts Intemational, 52/06b, 3327.

Thouless, R. H. (1931). Phenomenal regression to the real object. British Journal of Psychology, 22, 1-30.

WEBER, E. H. (1978). The sense of touch (H. E. Ross, Ed., and Trans.) London: Academic Press. (Original work published 1834)

WELCH, R. B., \& WARREN, D. H. (1981). Immediate perceptual response to intersensory discordance. Psychological Bulletin, 88, 638-667.

\section{NOTES}

1. Although there is no definite proof in this experiment that vision was successfully eliminated, the results of the control conditions in both Experiments 2 and 3 serve as solid confirmation. As will be seen, in the conditions in which the subjects were denied vision with the same blindfold and haptic volumetric cues were eliminated by lifting objects via a string, there was no size-weight illusion.

2. Subsequent analyses reveal that no appreciable differences are found when geometric means are employed.

(Manuscript received March 19, 1992; revision accepted for publication August 18, 1992.) 